Research Article

\title{
Chaotic Dynamics of Cage Behavior in a High-Speed Cylindrical Roller Bearing
}

\author{
Long Chen, Xintao Xia, Haotian Zheng, and Ming Qiu \\ School of Mechatronics Engineering, Henan University of Science and Technology, Luoyang 471039, China \\ Correspondence should be addressed to Long Chen; haustchenlong@163.com
}

Received 7 August 2015; Revised 14 December 2015; Accepted 15 December 2015

Academic Editor: Jussi Sopanen

Copyright ( 2016 Long Chen et al. This is an open access article distributed under the Creative Commons Attribution License, which permits unrestricted use, distribution, and reproduction in any medium, provided the original work is properly cited.

\begin{abstract}
This paper presents a mathematical model to investigate the nonlinear dynamic behavior of cage in high-speed cylindrical bearing. Variations of cage behavior due to varying cage eccentricity and cage guidance gap are observed. Hydrodynamic behavior in cage contacts is taken into consideration for a more realistic calculation of acting forces owing to high working speed. Analysis of realtime cage dynamic behavior on radial plane is carried out using chaos theory based on the theoretical and mathematical model established in the paper. The analytical results of this paper provide a solid foundation for designing and manufacturing of highspeed cylindrical roller bearing.
\end{abstract}

\section{Introductions}

Radial cylindrical roller bearings are designed to carry radial loads and be applied under high-speed conditions. Usually, cylindrical roller bearings may be obtained as a unit, which includes two steel rings each of which having a hardened raceway on which hardened cylindrical rollers roll. The rollers are usually held in an angularly spaced relationship by a cage. The cage is made from machined brass or pressed steel. Brass cage is widely used in high-speed application.

There are normally two methods to distinguish the type of cylindrical roller bearings. One is classified by arrangement of the ribs. Depending on the type of bearing, either the inner or the outer ring has two roller guiding ribs. The other is classified by the types of cage guidance. There are three types of cage guidance, as briefly demonstrated in Figure 1. They are outer-ring-rib guidance (Figure 1(a)), inner-ringrib guidance (Figure 1(b)), and roller guidance (Figure 1(c)), respectively. The weight of the cage acts on rollers directly of roller guidance bearing and it acts on inner/outer ring, respectively, of inner/outer guidance bearing when it is mounted horizontally in most common application.

As shown in Figure 1, four kinds of gaps between components can be found in the section. They are radial clearance $\left(G_{r}\right)$, roller gap $(\Delta)$, cage guidance gap $\left(C_{r}\right)$, and cage axial gap $\left(C_{a}\right)$, respectively. These gaps are defined as the maximum possible displacements between relative components in radial/axial direction. There is another important variable named pocket clearance $(T)$ in rolling bearing design, and it can be observed from Figure 2. Obviously, pocket clearance can be defined by the difference between cage pocket diameter and roller diameter. The cage pocket diameter has to be optimized to avoid faster wear of cage in terms of better lubrication film forming and decreased roller-cage bridge impact forces.

According to the general design guidelines of rolling bearings, the value of cage axial gap $\left(C_{a}\right)$ is larger than roller axial gap $(\Delta)$, the value of pocket clearance $(T)$ is larger than cage guidance gap $\left(C_{r}\right)$, and the value of cage guidance gap $\left(C_{r}\right)$ is larger than bearing radial clearance $\left(G_{r}\right)$ usually. These rules ensure that the rollers contact with raceways directly. The rollers' skewing can be adjusted by ring ribs according to the rules. Then the cage is in a certain state of being "free" in the bearing.

When cylindrical bearings operate at a high speed, they generate vibrations and noise. The principal forces, which drive these vibrations, are time varying nonlinear contact forces, which exist among the various components of the bearings: rings, rollers, and cage. In the last decades, a lot of efforts have been devoted to studying the stability 


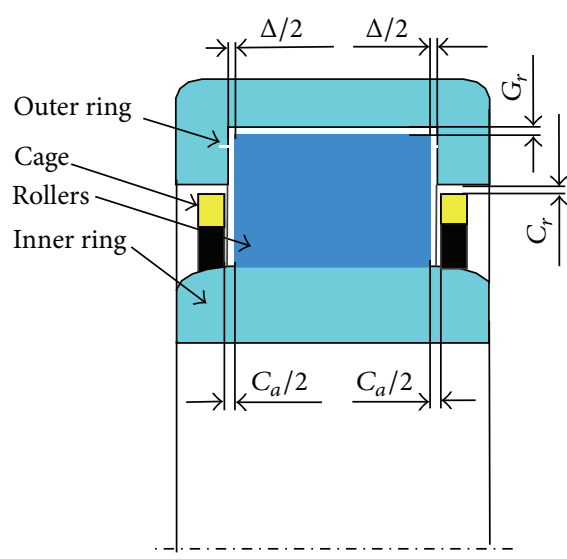

(a) Outer-ring-rib guidance

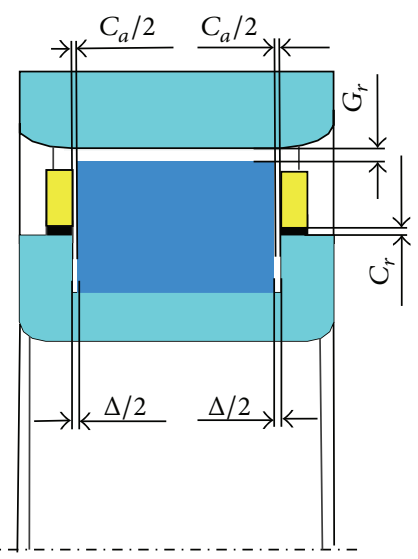

(b) Inner-ring-rib guidance

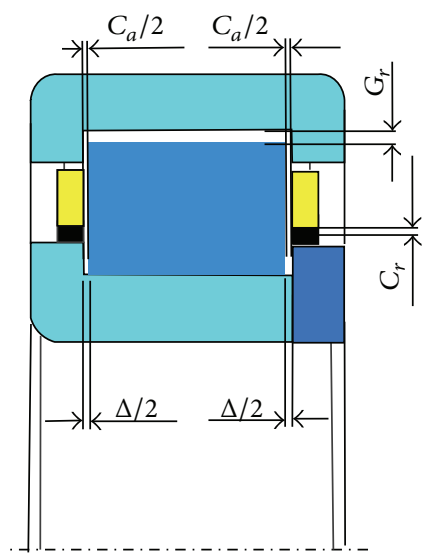

(c) Roller guidance

FIGURE 1: Cage guidance in cylindrical roller bearings.

and nonlinear dynamic behavior of flexible rotor bearings. Obviously, one of the most important mechanical elements to be taken into account is bearings due to their large influence on the dynamic behavior of rotating machinery (Tiwari et al. [1], Adam Jr. [2]).

Many researchers developed special technique on dynamic behavior considering details of geometric parameters and applicable conditions of rolling bearings. Aktürk et al. [3] and Upadhyay et al. [4] presented theoretical investigations of varying preload, the influence of the number of the balls, and ball diameters on vibration characteristics of a rotor bearing system. Aktürk [5] and Harsha et al. [6] researched the effect of surface waviness on vibrations of ball bearings. Sopanen and Mikkola [7] and Upadhyay et al. [8] investigated dynamic behaviors of high-speed rotation with localized and distributed defects. Harsha [9] and Villa et al. [10] presented a nonlinear dynamic analysis of a flexible unbalanced rotor supported by ball bearings.

The importance of energy efficiency has been increasing and has become a quality criterion for bearing producers and users in recent years. Hence, more and more researchers drew their attention on dynamic behaviors of the cage in rolling bearing. Houpert [11] developed a simulation software to simulate cage behavior and carried out relative experimental validation. Harsha [12] analyzed the nonlinear dynamics of ball bearings due to cage run-out and varying number of balls. He presented the results in the form of fast Fourier transformations (FFT) and phase trajectories. It is implied from the obtained FFT that due to the nonuniform spacing the ball passage frequency is modulated with the cage frequency. Bercea et al. [13] and Sakaguchi and Harada [14] investigated cage behavior of tapered roller bearings and made a comparison between numerical and experimental results.

In this paper, a theoretical investigation considering all possible contacts of cage is conducted to observe its dynamic behavior. Owing to the investigation object applied in highspeed working condition, hydrodynamics in the contacts is taken into consideration for a more realistic calculation of acting forces by simulation model with the hypothesis of enough lubrication to supply all the contacts. Brass is chosen as the raw material of the cage in the paper considering the working speed of the bearing. Brass cages are machined by lathe and drilling in processing technique in effect. This causes uneven quality distribution and error of the cage roundness. Then different cage eccentricity and cage gap draw our attention and they are analyzed. In addition, lubrication dynamic viscosity exponent is another key factor in the application of high-speed bearings. Influence of different lubrication dynamic viscosity exponents is investigated as well. Chaos theory is applied for analyzing above-mentioned parameters. Both Poincaré map and bifurcation diagram are obtained and the key factor which influences the dynamic behavior is found.

\section{Static Displacements of the Cage}

The bearing under study has the outer ring fixed to a rigid support and the inner ring fixed rigidly to the shaft. A constant vertical radial load acts on the bearing, and it shifts the inner ring to the outer ring through rollers. The static radial displacement is influenced by many factors, such as clearance, load, and number of the rollers.

2.1. Displacements of Different Guidance Types. As illustrated in the instructions, different guidance types mean different cage structure and different contact position. Then different displacement caused by different guidance type is described first here.

2.1.1. Outer-Ring-Rib-Guidance Bearing. As can be seen in Figure 1(a), radial gap between cage outer diameter and inner diameter of outer ring rib is the only moveable space for outer-ring-rib-guidance cylindrical roller bearing. Then the displacement is confirmed by cage guidance gap $\left(C_{r}\right)$ and it can be given by

$$
Y_{c o}=\frac{C_{r}}{2} .
$$




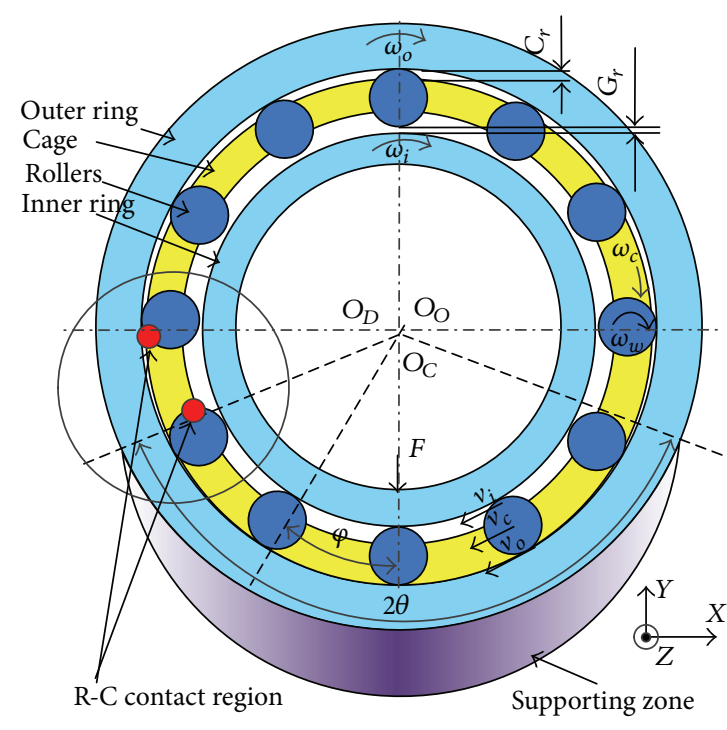

(a) Schematic diagram

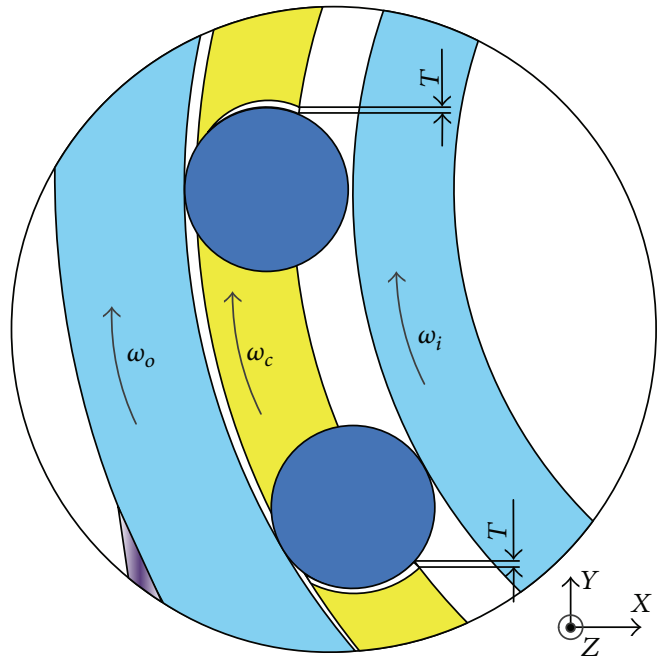

(b) Pocket clearance

FIGURE 2: Schematic diagram and pocket clearance in a cylindrical roller bearing.

2.1.2. Inner-Ring-Rib-Guidance Bearing. Inner ring displacement of inner-ring-rib-guided bearing is caused by the clearance $\left(G_{r}\right)$, cage guidance gap $\left(C_{r}\right)$, and the elastic deformation $\left(\delta_{r}\right)$ in the contact lines of the raceways and the rollers. The value of elastic deformation depends on the value of the load and the number of the rollers under load. Then supporting zone, as can be seen in Figure 2(a), should be confirmed firstly. The angular extent of the supporting zone is determined by the radial clearance $\left(G_{r}\right)$ of a cylindrical roller bearing such as

$$
2 \theta=\cos ^{-1} \frac{G_{r}}{2 \delta_{r}} .
$$

Elastic deformation between the raceway and roller shows a nonlinear relation, which is obtained by using the Hertzian theory [15]. The local Hertzian contact force and deflection relationship for a bearing may be written as

$$
\begin{aligned}
Q_{i} & =K_{i} \delta_{i}{ }^{10 / 9}, \\
Q_{o} & =K_{o} \delta_{o}{ }^{10 / 9} .
\end{aligned}
$$

The local Hertzian contact forces in (3) are determined by external load $(F)$, number of rollers in supporting zone $(N)$, and the radial clearance $\left(G_{r}\right)$. The calculation method is a very standardized procedure and will not be described here. The total normal approach between two raceways under load separated by a roller is the sum of the approaches between the roller and each raceway. Hence

$$
\delta_{r}=\delta_{r i}+\delta_{r o} .
$$

Then the radial displacement of the cage can be obtained from above equations as

$$
Y_{c i}=\delta_{r}+\frac{1}{2} G_{r}+\frac{1}{2} C_{r}
$$

2.1.3. Roller Guidance. The normal approach between two raceways under load influences the cage displacement directly in this kind of guidance type. The cage displacement in roller-guidance bearing can be given as

$$
Y_{c w}=\delta_{r}+\frac{1}{2} G_{r}+T .
$$

2.2. Cage Displacement Caused by Rotating. Bearings are used to bear various kinds of loads while keeping a shaft rotating. Rollers are subjected to dynamic loading due to speed effects. As a general case it will be initially assumed that both inner and outer rings are rotating in a bearing, as illustrated in Figure 2. Consequently,

$$
\begin{aligned}
& v_{i}=\frac{1}{2} \omega_{i} d_{i}, \\
& v_{o}=\frac{1}{2} \omega_{o} d_{o} .
\end{aligned}
$$

Then the cage speed, or the speed of rotation of the set of rollers around the origin, is

$$
\omega_{c}=\frac{1}{2}\left[\omega_{i}\left(1-\frac{d_{w}}{d_{m}}\right)+\omega_{o}\left(1+\frac{d_{w}}{d_{m}}\right)\right] .
$$

The centrifugal force caused by the $j$ th roller is calculated as

$$
F_{j_{\text {cen }}}=m_{j} \frac{1}{2} \omega_{c}^{2} d_{m} .
$$

According to design guidelines of rolling bearings, the value of $C_{a}$ is larger than that of $\Delta$, the value of $T$ is larger than that of $C_{r}$, and the value of $C_{r}$ is larger than that of $G_{r}$ generally. Thus the rollers' centrifugal forces are acting 


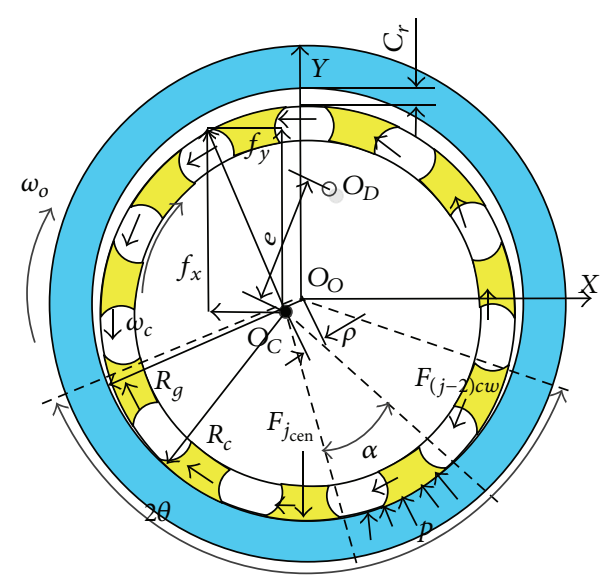

FIGURE 3: Forces generated by motion on the cage.

on outer raceway. Subsequently, the centrifugal forces of the rollers cause larger contact deformation. It can be added to the original deformation on the outer raceway as

$$
\delta_{r o}=\left(\frac{F_{\mathrm{cen}}+Q_{o}}{K_{o}}\right)^{9 / 10} .
$$

Substituting (10) into (4), equations (5) and (6) will change along with (4). According to different expressions of cage displacements, both inner ring guidance and roller guidance bearings will generate complex dynamic displacements when they are operating at high speeds. Then we can draw our conclusion that both inner ring guidance and roller guidance are not applicable under high-speed conditions. Outer-ringrib-guidance bearing is chosen as the research object in the following analysis naturally.

\section{Physical Model}

The cage has two kinds of contacts in a rotating outer-ringguided cylindrical bearing. One is the cage-rib contact and the other is the roller-pocket contact. The forces generated by the contacts are marked in Figure 3. Cage-rib contact status is determined by the relative rotational speed $\left(\omega_{c o}\right)$ and the cage guidance gap $\left(C_{r}\right)$. The cage has physical contact with guiding ring in the starting phase owing to a low relative speed. However, the cage and the rib are separated by oil film eventually with the increasing of relative speed. Then cage-rib contact is considered as a fluid-structure interaction problem. Similarly, roller-cage contact status is determined by rotational speed of the roller around its own axil $\left(\omega_{c w}\right)$ and the pocket clearance $(T)$. The cage and the rollers are separated by oil film with the increasing of speed too. Note that the cage is driven by the rollers in supporting zone and the rollers are driven by cage in nonloaded zone. The driving forces by rollers on the cage can be seen in Figure 3 as well. Different driving mode of the rollers in different force directions causes impact loads on the cage. The impact between the cage and the roller in a very short time results in large impact forces.
3.1. Instantaneous Motion Forces. Different contacts cause different motion forces and there is no strong relationship among the motion forces; then linear superposition method can be adopted for calculating motion forces on the cage.

The relative rotational velocity of the cage to the rib is defined as

$$
\omega_{c o}=\omega_{c}-\omega_{o} .
$$

And the cage guidance gap determined by cage diameter and rib can be expressed as

$$
C_{r}=d_{g}-d_{c} .
$$

Brass cage is usually machined from centrifugally cast tubing, and then nonuniform distribution of the mass causes static imbalance $(e)$ of the cage. Nondimensional eccentricity of the cage to cage guidance gap can be given as

$$
\rho=\frac{e}{C_{r}} .
$$

Spiridon [16] established an elastic system with two degrees of freedom (DOF) that considered both cageroller stiffness $\left(K_{c w}\right)$ and cage structural stiffness $\left(K_{c}\right)$ for cage/roller contact. As described in Figure 4, the dynamic equilibrium condition written for each of the two masses provides the following set of two differential equations:

$$
\begin{array}{r}
K_{c w}\left(\delta_{w}-\delta_{c}\right)^{10 / 9}+M_{w} \frac{d^{2} \delta_{w}}{d t^{2}}=0, \\
-K_{c w}\left(\delta_{w}-\delta_{c}\right)^{10 / 9}+K_{c} \delta_{c}+M_{c} \frac{d^{2} \delta_{w}}{d t^{2}}=0 .
\end{array}
$$

$K_{c w}$ can be derived from the Hertzian contact theory, whereas $K_{c}$ can be obtained analytically. The cage-roller stress $\left(Q_{c w}\right)$ can be obtained from (3) and its variation trend for the specified roller is shown in Figure 5 when the bearing is rotating.

Impact force of cage/roller contact can be given as

$$
\begin{aligned}
& f_{h x}=Q_{c w}\left[\sin \omega_{c o} t+\sin \left(\pi-\omega_{c o} t\right)\right] \cos \theta, \\
& f_{h y}=Q_{c w}\left[\sin \omega_{c o} t-\sin \left(\pi-\omega_{c o} t\right)\right] \sin \theta .
\end{aligned}
$$

As for the cage-rib dynamic model, it can encompass radial-plane orbital cage motion with 2-DOF equations as well. According to Figure 4, motion equation can be expressed as

$$
\begin{aligned}
M_{D} \ddot{O}_{D}= & -2 K_{s}\left(O_{C}-O_{O}\right)-2 B_{s}\left(\dot{O}_{C}-\dot{O}_{O}\right) \\
& +2 M_{D} \rho g e_{y} \\
& -F_{s}\left(X_{D}, \dot{X}_{D}, \ddot{X}_{D}, Y_{D}, \dot{Y}_{D}, \ddot{Y}_{D}\right) \\
M_{C} \ddot{O}_{C}= & -K_{s}\left(O_{C}-O_{O}\right)-B_{s}\left(\dot{O}_{C}-\dot{O}_{O}\right)+f_{e x} \cdot e_{x} \\
& +f_{e y} \cdot e_{y}-M_{O} \rho g e_{y} .
\end{aligned}
$$




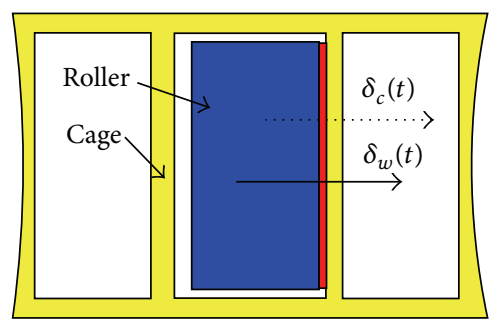

(a) Cage/roller contact

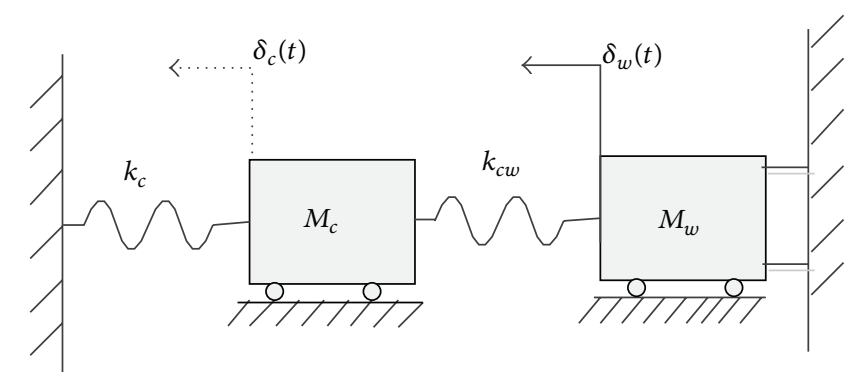

(b) Model of cage/roller contact

FIGURE 4: A 2-DOF model of cage/roller contact of the $j$ th roller.

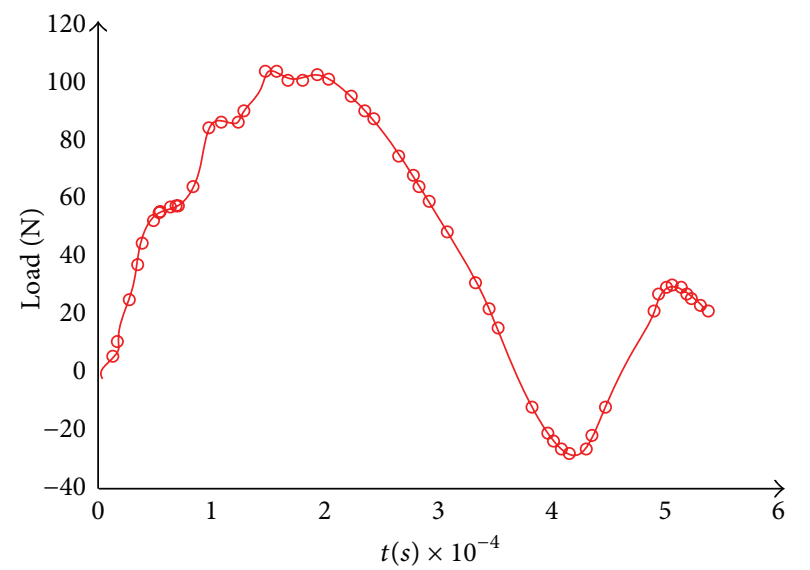

FIGURE 5: Demonstration on variation trend of cage-roller contact force.

The position vector, velocity vector, and acceleration vector of the unbalanced gravitational center of the cage can be given as

$$
\begin{aligned}
& O_{D}=\left(X_{c}+\rho \cos \omega t\right) \cdot e_{x}+\left(Y_{c}+\rho \sin \omega t\right) \cdot e_{y}, \\
& \dot{O}_{D}=\left(\dot{X}_{c}+\rho \cos \omega t\right) \cdot e_{x}+\left(\dot{Y}_{c}+\rho \sin \omega t\right) \cdot e_{y}, \\
& \ddot{O}_{D}=\left(\ddot{X}_{c}+\rho \cos \omega t\right) \cdot e_{x}+\left(\ddot{Y}_{c}+\rho \sin \omega t\right) \cdot e_{y} .
\end{aligned}
$$

In the same way, the position, velocity, and acceleration vector of geometry center of the cage are

$$
\begin{aligned}
& \mathrm{O}_{c}=X_{c} \cdot e_{x}+Y_{c} \cdot e_{y}, \\
& \dot{O}_{c}=\dot{X}_{c} \cdot e_{x}+\dot{Y}_{c} \cdot e_{y}, \\
& \ddot{O}_{c}=\ddot{X}_{c} \cdot e_{x}+\ddot{Y}_{c} \cdot e_{y} .
\end{aligned}
$$

Similarly, using above-mentioned exhibition, the position, velocity, and acceleration vector of the center of the rib are

$$
\begin{aligned}
& O_{o}=X_{o} \cdot e_{x}+Y_{o} \cdot e_{y}, \\
& \dot{O}_{o}=X_{o} \cdot e_{x}+\dot{Y}_{o} \cdot e_{y}, \\
& \ddot{O}_{o}=X_{o} \cdot e_{x}+\ddot{Y}_{o} \cdot e_{y} .
\end{aligned}
$$

Substituting (15), (16), and (17) into (14), then the following two equations described in $x-y$ plane can be obtained:

$$
\begin{aligned}
& M_{D} \ddot{X}_{O} \\
&=-2 K_{s}\left(X_{C}-X_{O}\right)-2 B_{s}\left(\dot{X}_{C}-\dot{X}_{O}\right) \\
&+2 M_{D} \rho \omega_{c-o}^{2} \cos \omega_{c-o} t-K_{x y} Y_{D}-K_{x y}^{\prime} Y_{D}^{3}, \\
& M_{D} \ddot{Y}_{O} \\
&=-2 K_{s}\left(Y_{C}-Y_{O}\right)-2 B_{s}\left(\dot{Y}_{C}-\dot{Y}_{O}\right) \\
&+2 M_{D} \rho \omega_{c-o}^{2} \sin \omega_{c-o} t-K_{x y} X_{D}-K_{x y}^{\prime} X_{D}^{3} \\
&-2 M_{D} g, \\
& M_{C} \ddot{X}_{C}-f_{e x}-K_{s}\left(X_{O}-X_{C}\right)-B_{s}\left(\dot{X}_{O}-\dot{X}_{C}\right)=0, \\
& M_{C} \ddot{Y}_{C}-f_{e y}-K_{s}\left(Y_{O}-Y_{C}\right)-B_{s}\left(\dot{Y}_{C}-\dot{Y}_{O}\right) \\
&=- M_{C} g .
\end{aligned}
$$

Considering (20)-(21), the results can be obtained as

$$
\begin{aligned}
f_{e x}= & v_{1}^{2}\left(\ddot{x}_{O}-\ddot{x}_{C}\right)-v_{2}\left(\dot{x}_{O}-\dot{x}_{C}\right)-v_{3}\left(x_{O}-x_{C}\right), \\
f_{e y}= & v_{2}^{2}\left(\ddot{y}_{O}-\ddot{y}_{C}\right)-v_{2}\left(\dot{y}_{O}-\dot{y}_{C}\right)-v_{3}\left(y_{O}-y_{C}\right) \\
& +K_{f_{d}} f_{d} .
\end{aligned}
$$

Here, $x_{O}, y_{O}, x_{C}$, and $y_{C}$ are nondimensional form of rib center $\left(X_{O}, Y_{O}\right)$ and cage center $\left(X_{C}, Y_{C}\right)$, and they are obtained by original value divided by $C_{r}$. The other factors in (22) can be expressed as

$$
\begin{aligned}
& v_{1}=\frac{2\left(M_{D} \omega_{c o} B\right)^{2}}{W}, \\
& v_{2}=2 K_{s} B, \\
& v_{3}=\frac{2 K_{s}^{3 / 2} B}{M_{D}^{1 / 2}} \omega_{c o} .
\end{aligned}
$$


When the linear superposition method is adopted to contact forces of the cage in $x-y$ plane, the total force on different directions can be given as

$$
\begin{aligned}
& f_{x}=f_{e x}, \\
& f_{y}=f_{a y}+f_{e y} .
\end{aligned}
$$

3.2. Hydrodynamic Fluid-Film Force. Cage-rib contact is a fluid-structure interaction problem with bearings rotating at a high speed. The main supporting force generated by lubricant squeeze effects can be approximated to a shortwidth journal bearing theory, as illustrated in Figure 3. Then dynamic-deviation interactive force between cage and rib can be described as a continuous function of cage position and velocity components. Reynolds lubrication equation (RLE) provides the basis of lubrication theory [15], and the force given on the cage by the film is expressed as

$$
\begin{gathered}
\frac{\partial}{\partial \alpha}\left[(1+\varepsilon \cos \alpha)^{3} \frac{\partial p}{\partial \alpha}\right]+R_{c}^{2} \frac{\partial}{\partial x}\left[(1+\varepsilon \cos \alpha)^{2} \frac{\partial p}{\partial y}\right] \\
\quad=-6 \mu\left(\frac{R_{c}}{C_{r}}\right)^{2}\left[\left(\omega_{c o}-2 \dot{\varphi}\right) \varepsilon \sin \alpha-2 \varepsilon \cos \alpha\right] .
\end{gathered}
$$

And

$$
p(\alpha)=P_{0}+\frac{\omega_{c o}}{C_{r}^{3}}\left[\frac{l_{c}^{2}}{4}-y^{2}\right] \frac{\varepsilon \sin \alpha}{1+\varepsilon \cos ^{3} \alpha} .
$$

Solutions to the RLE are a nonlinear function of displacement and angle at cage center. Hydrodynamic fluid-film force can be written as follows:

$$
\begin{aligned}
f_{e} & =\mu R_{c} l_{c}\left(\frac{l_{c}}{C_{r}}\right)^{2}\left[\left(\omega_{c o}-2 \dot{\alpha}\right) \frac{\varepsilon^{2}}{\left(1-\varepsilon^{2}\right)^{2}}\right. \\
& \left.+\frac{\pi}{2} \frac{\left(1+2 \varepsilon^{2}\right) \dot{\varepsilon}}{\left(1-\varepsilon^{2}\right)^{5 / 2}}\right], \\
f_{\alpha} & =\mu R_{c} l_{c}\left(\frac{R_{c}}{C_{r}}\right)^{2} \\
& \cdot\left\{\left[\left(\omega_{c o}-2 \alpha\right) \frac{\pi \varepsilon}{4\left(1-\varepsilon^{2}\right)^{3 / 2}}+\frac{2 \varepsilon \dot{\varepsilon}}{\left(1-\varepsilon^{2}\right)^{2}}\right]\right. \\
& \left.+2 R_{c} l_{c} p_{0}\right\} .
\end{aligned}
$$

The fluid-film force can also be represented as two component forces in horizontal and vertical force equilibrium:

$$
\begin{aligned}
& f_{x}=f_{e} \cdot \cos \alpha+f_{\alpha} \cdot \sin \alpha, \\
& f_{y}=f_{e} \cdot \sin \alpha-f_{\alpha} \cdot \cos \alpha .
\end{aligned}
$$

3.3. Equilibrium of the Equations. In hydrodynamic fluidfilm bearing, the fluid supported pressure is generated normally by motion of the cage and depends on the dynamic viscosity exponent of the lubricating fluid. For the cage structure and coordination please refer to Figure 6. The generated forces can be expressed as

$$
\begin{aligned}
f_{e} \cdot \cos \alpha+f_{\alpha} \cdot \sin \alpha= & v_{1}^{2} \ddot{x}_{C}-v_{2}\left(\dot{x}_{O}-\dot{x}_{C}\right) \\
& -v_{3}\left(x_{O}-x_{C}\right), \\
f_{e} \cdot \sin \alpha-f_{\alpha} \cdot \cos \alpha= & v_{1}^{2} \ddot{y}_{C}-v_{2}\left(\dot{y}_{O}-\dot{y}_{C}\right) \\
& -v_{3}\left(y_{O}-y_{C}\right)+B_{f_{d}} f_{d} \\
& +f_{i y} .
\end{aligned}
$$

Displacement relations between the centers of cage and rib are

$$
\begin{aligned}
& x_{c}=x_{o}+\varepsilon \cos \varphi, \\
& y_{c}=y_{o}+\varepsilon \sin \varphi .
\end{aligned}
$$

Similarly for the velocity

$$
\begin{aligned}
& \dot{x}_{c}=\dot{x}_{o}+\dot{\varepsilon} \cos \varphi-\varepsilon \sin \varphi \cdot \dot{\varphi}, \\
& \dot{y}_{c}=\dot{y}_{o}+\dot{\varepsilon} \sin \varphi-\varepsilon \cos \varphi \cdot \dot{\varphi} .
\end{aligned}
$$

Substituting (27), (30), and (31) into (29), integration of equation can be obtained:

$$
\begin{aligned}
& \ddot{\varepsilon}\left(1-\varepsilon^{2}\right)^{3}+\dot{\varepsilon}\left[v_{2}\left(1-\varepsilon^{2}\right)^{3}\right. \\
& \left.-v_{1}^{\prime} \frac{\pi}{2}\left(1+2 \varepsilon^{2}\right)\left(1-\varepsilon^{2}\right)^{1 / 2}\right]-v_{2} \varepsilon \sin 2 \alpha \cdot \dot{\alpha}(1 \\
& \left.-\varepsilon^{2}\right)^{3}+v_{3} \varepsilon\left(1-\varepsilon^{2}\right)^{3}-\mu \cos 2 \alpha-v_{1}^{\prime}\left(\omega_{c o}-2 \dot{\alpha}\right) \\
& \cdot \varepsilon^{2}\left(1-\varepsilon^{2}\right)=0 \\
& \ddot{\alpha}\left[\varepsilon\left(1-\varepsilon^{2}\right)^{2}+\varepsilon\left(1-\varepsilon^{2}\right)^{2} 2 \mu \sin 2 \alpha \cdot\left(1-\varepsilon^{2}\right)^{2}\right] \\
& +\dot{\alpha}\left[2 \dot{\varepsilon}\left(1-\varepsilon^{2}\right)^{2}+v_{2} \varepsilon\left(1-\varepsilon^{2}\right)^{2}\right] \\
& +v_{1}^{\prime}\left\{\left[\left(\omega_{c o}-2 \alpha\right) \pi \varepsilon\left(1-\varepsilon^{2}\right)^{1 / 2}+2 \varepsilon \dot{\varepsilon}\right]\right. \\
& \left.+2 R_{c} l_{c} P_{0}\right\}=0 \text {. }
\end{aligned}
$$

Equation (32) can be solved by modified Newmark- $\beta$ method to obtain the displacement and velocity of the cage.

\section{Chaos Analyses}

Chaos Theory is a new way to analyze complexity which Henri Poincaré studied as the possibility of forecasting. A small variation in the initial conditions can generate a different set of results/solutions [17].

A bearing typed as NU2310 is chosen to analyze chaotic response. Tomoya and Kaoru [18] tested the dynamic cage displacements of this bearing and the maximum displacements can be used to verify the validity of theoretical analysis. 


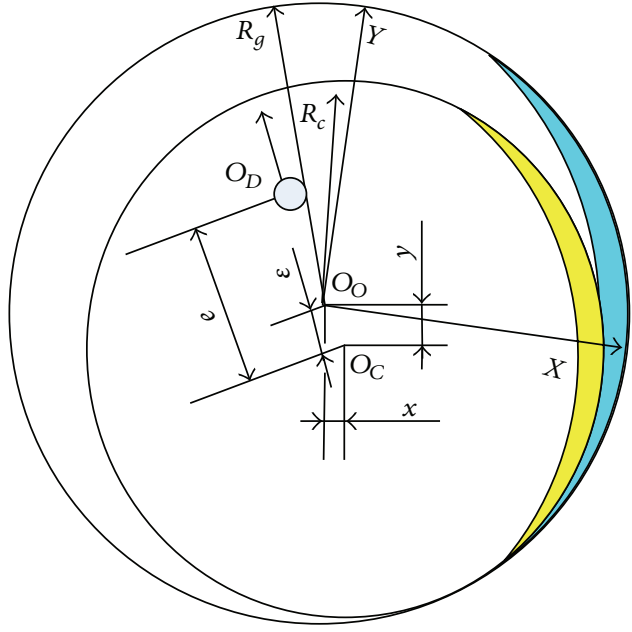

(a) Cage-rib contact

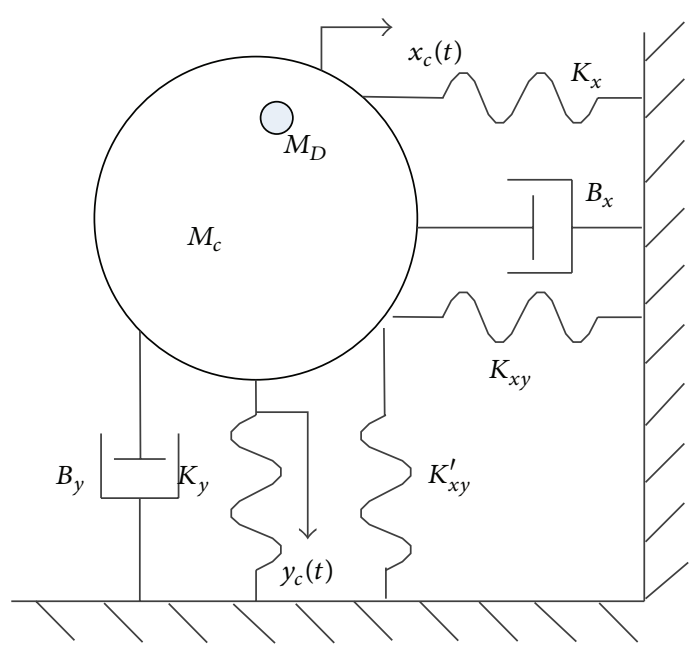

(b) Cage-rib contact model

FIGURE 6: 2-DOF model of cage/rib contact.

The structural specifications of the bearing are listed in Table 1.

The cage of the bearing is made of machined brass DIN $\mathrm{CuZn} 40 \mathrm{~Pb} 2 \mathrm{~F} 43$ (GB ZCuZn38Mn2Pb2), and the rings of the bearing are made of through-harden rolling bearing steel DIN 100Cr6 (SAE 52100/GB GCr15). The cage is guided by the outer-ring rib. Both static load rating and dynamic load rating are $186 \mathrm{KN}$. According to the recommendation of the manufacturer, reference speed rating is $6700 \mathrm{rpm}$ and limited speed rating is $12000 \mathrm{rpm}$. Then the weight of the cage and damping and stiffness parameters can be obtained according to the relative parameters in Table 1 . The displacement in $x$ direction is zero and the displacement in $y$ direction is determined by the cage guidance gap according to (1). Bifurcation diagram of $X_{C}$ versus spin speed increases with different cage guide gaps, cage eccentricities, and dynamic viscosity exponent of lubrication oil. The change curves are described in Figure 7, respectively. All three cases are simulated and variation parameters of the cases are listed in Table 2. An interesting phenomenon that all the cases have two bifurcation zones and two steady zones is found in all curves. In relative low rotation speed, transient responses can be found. The dynamic response of cage center goes into the first ordered stage after the end of the resonance. Then the first bifurcation response occurs. The second ordered status arises after the first bifurcation ends and then the curve goes into the second bifurcation. Figure 7 (a) compares the bifurcation diagrams of cage with different guidance gaps. The first chaotic response zone of the bearing with smaller guidance gap (case 1a) is from $\omega_{c o}=9100 \mathrm{rpm}$ to $\omega_{c o}=12000 \mathrm{rpm}$, and it is from $\omega_{c o}=7100 \mathrm{rpm}$ to $\omega_{c o}=22000 \mathrm{rpm}$ for the bearing with larger guidance gap (case $1 \mathrm{~b}$ ). The second chaotic response zone of the bearing with smaller guidance gap is from $\omega_{c o}=25500 \mathrm{rpm}$, and it is from $\omega_{c o}=26000 \mathrm{rpm}$ for the bearing with larger guidance gap. Chaotic response zone is wider when the gap is larger. Figure $7(\mathrm{~b})$ shows bifurcation diagrams of cage with different eccentricities. The first chaotic zone of case $2 \mathrm{a}$ is from $\omega_{c o}=7600 \mathrm{rpm}$ to $\omega_{c o}=14000 \mathrm{rpm}$, and it is from $\omega_{c o}=7800 \mathrm{rpm}$ to $\omega_{c o}=13000 \mathrm{rpm}$ of case $2 \mathrm{~b}$. The second chaotic zone of case $2 \mathrm{a}$ is from $\omega_{c o}=22500 \mathrm{rpm}$, and it is from $\omega_{c o}=19800 \mathrm{rpm}$ of case $2 \mathrm{~b}$. Chaotic response zone increases with eccentricity. In addition, transient oscillation is more drastic when comparing case la and case 2a. Figure 7(c) depicts bifurcation diagrams of cage with different dynamic viscosity exponents. The first chaotic zones of case $3 \mathrm{a}$ and case $3 \mathrm{~b}$ are from $\omega_{c o}=7650 \mathrm{rpm}$ to $\omega_{c o}=13400 \mathrm{rpm}$. The second chaotic zones of case $3 \mathrm{a}$ and case $3 \mathrm{~b}$ are from $\omega_{c o}=$ $21000 \mathrm{rpm}$. The change trend of case 3 a curve is approaching to that of case 3b. But the variation curve of cage with VG78 is lower than that with VG56 due to higher dynamic viscosity exponent.

The first bifurcation point $Y_{C}$ versus spin speed zooming is shown in Figure 8. Fluctuation can be found even in steady zone owing to the impact forces between roller and cage pockets. In low-speed zone, the wave frequency is low and it increases with rotational speed. The difference of steady zone range is shown more clearly in the enlarged view. Bifurcation diagrams of each case are different obviously and this denotes that the position of $X_{C}$ varies timely as well.

Cage response behaviors of chaos which are subjected to the cases in Table 2 at $\omega=8500$ RPM, Poincaré section points of $Y_{C}$ displacement in vertical direction, and time history for 4th revolution to 4000th revolution are shown in Figure 9.

\section{Conclusion and Discussion}

Rolling bearing life is typically calculated on the basis of its load ratings relative to the applied loads and the requirements regarding bearing life and reliability. Both dynamic load rating and rating life equations neglect cage design. Actually, the cage design has evolved to bear higher and higher load by incorporating more rollers, and to lower costs by adopting 


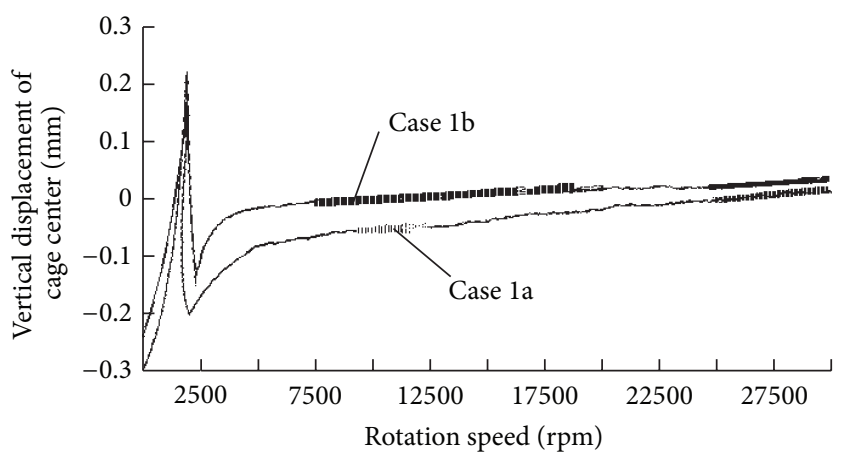

(a) Different cage guidance gaps

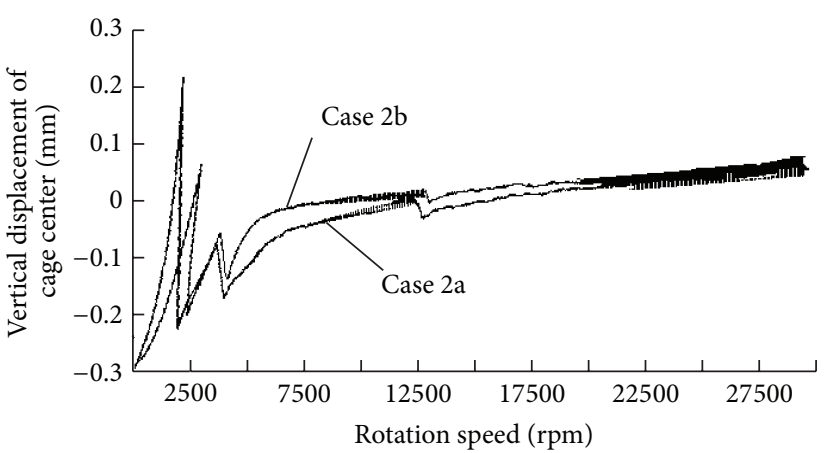

(b) Different eccentricities

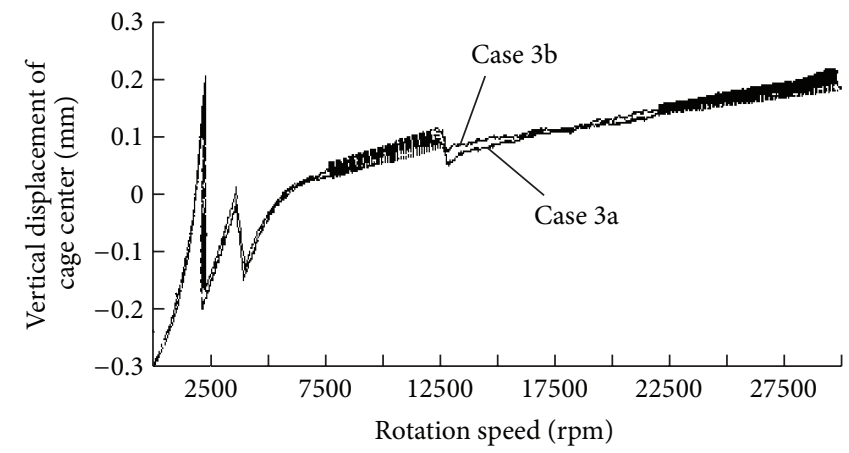

(c) Different dynamic viscosity exponents

FIgURE 7: Bifurcation diagram points, $X_{C}$ versus spin speed.

TABLE 1: Specifications of the bearing structure and size.

\begin{tabular}{lccccccccc}
\hline Bearing type & $\begin{array}{c}\text { Bore } \\
\mathrm{mm}\end{array}$ & $\begin{array}{c}\text { Boundary size } \\
\text { Outside } \\
\mathrm{mm}\end{array}$ & $\begin{array}{c}\text { Width } \\
\mathrm{mm}\end{array}$ & Number & $\begin{array}{c}\text { Roller size } \\
\text { Diameter } \\
\mathrm{mm}\end{array}$ & $\begin{array}{c}\text { Length } \\
\mathrm{mm}\end{array}$ & $\begin{array}{c}\text { Rib diameter } \\
\text { mm }\end{array}$ & $\begin{array}{c}\text { Radial } \\
\mu \mathrm{m}\end{array}$ & $\begin{array}{c}\text { Pocket } \\
\mathrm{mm}\end{array}$ \\
\hline NU2310 & $\Phi 50$ & $\Phi 110$ & 40 & 13 & $\Phi 16$ & 27 & $\Phi 92.1$ & 40 \\
\hline
\end{tabular}

TABle 2: Case study.

\begin{tabular}{cccc}
\hline Case & $\begin{array}{c}\text { Guidance gap } \\
(\mathrm{mm})\end{array}$ & $\begin{array}{c}\text { Variation parameters } \\
\text { Eccentricity } \\
(\mathrm{mm})\end{array}$ & $\begin{array}{c}\text { Dynamic viscosity exponent } \\
(\mathrm{Pa} \cdot \mathrm{s})\end{array}$ \\
\hline $\mathrm{la}$ & 0.45 & 2.3 & VG56 \\
$\mathrm{lb}$ & 0.60 & 2.3 & VG56 \\
$\mathrm{2a}$ & 0.45 & 3.5 & VG56 \\
$\mathrm{2b}$ & 0.45 & 5.7 & VG56 \\
$3 \mathrm{a}$ & 0.45 & 4.2 & VG78 \\
$3 \mathrm{~b}$ & 0.45 & 4.2 & VG32 \\
\hline
\end{tabular}

different material and improve high-speed performance by using optimized geometry structure. This paper pays close attention to influence from cage on speed characteristics of cylindrical roller bearings. The most suitable cage guidance type is confirmed by mathematical derivation firstly. Two major factors originating from manufacturing process and one involved in applying process are considered to establish dynamical mathematical model of cage with appropriate cage guidance type. Analysis of real-time dynamic behavior of a cage on radial plane was carried out using chaos theory based on the theoretical mathematical model. The chaotic analysis is limited by the initial values of the system. Hence, the initial values are determined firstly in analytical process. Preliminary conclusions are listed as follows.

(1) There are two bifurcation zones of cage dynamic curves and the bearing should be away from these two zones in practice. The range of the 1st bifurcation zone is mainly influenced by the value of cage guidance gap and eccentricity, especially the cage guidance gap. The lubrication dynamic viscosity exponent has relatively little influence on cage dynamic.

(2) Numerical simulations show that the roller tends to accelerate/decelerate in switching process of supporting zone/loaded zone, which leads to single or multiple roller-cage bridge impacts. Impact force causes periodic small oscillation on the curves. Oscillation amplitude is determined by the pocket gap.

(3) Eccentricity of the cage causes dramatic transient responses in low rotational speed zone. The transient responses are easily tending to cause knocking and 


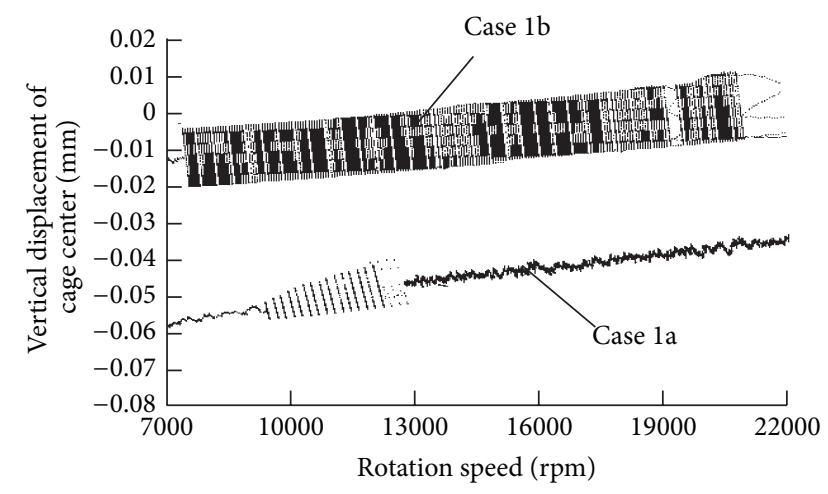

(a) Different cage guidance gaps

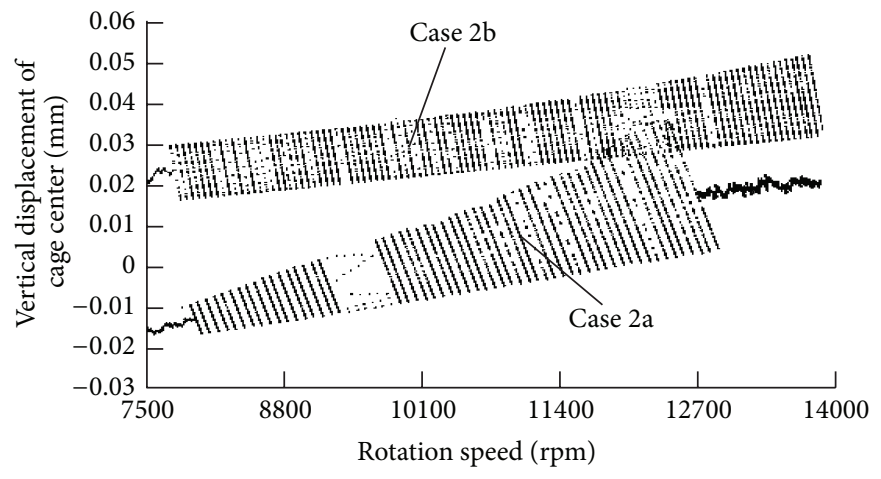

(b) Different eccentricities

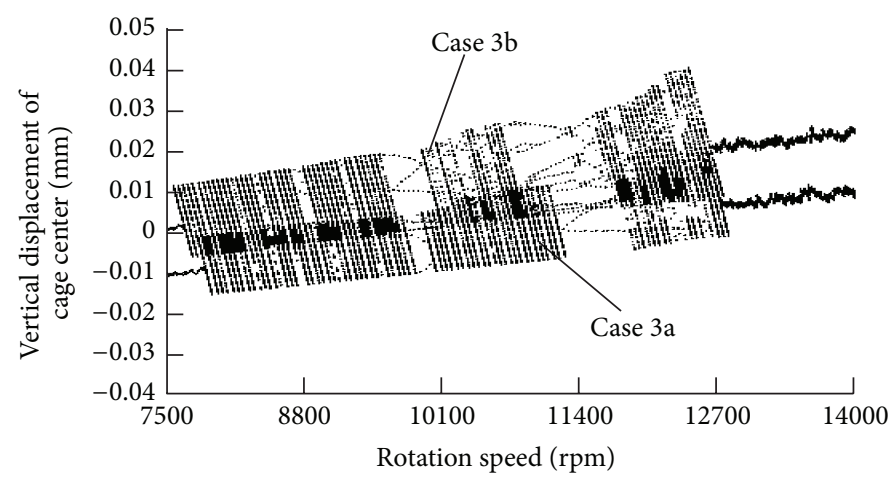

(c) Different dynamic viscosity exponents

FIgURE 8: The first bifurcation zone from Figure 7 in enlarged scale.

faster wear of cage pocket in terms of worse lubrication film forming. The steady zone is narrower when eccentricity increases. In addition, divergent trend can be found in the second bifurcation zone with lager eccentricity.

(4) According to the zooming of the first bifurcation zone, variations of any parameters cause totally different bifurcations. We can draw our conclusion that movement of cage is a chaotic motion.

(5) The Poincaré maps show that position and rotational speed of the occurrence bifurcation zones are very different with varied parameters.

(6) The change of fluid dynamic viscosity exponent will not affect the region of bifurcation due to the weak nonlinear of fluid film force.

Considering cage dynamics of high-speed cylindrical roller bearing, the values of cage guidance gap and eccentricity are crucial factors. From the point of view of the manufacturers, the very small cage guidance gap is difficult to obtain owing to the material (brass) of the cage used in highspeed bearing. The authors strongly suggest that the designer should minimize the gap within the confine of processing capacity. As for the cage eccentricity, dynamic balance of cage is widely used in ultra-high-speed bearings. Analyses from the paper show that cage eccentricity brings violent oscillation in low-speed zone, and oscillation amplitude in chaotic zone is higher with the increase of cage eccentricity. Hence, dynamic balance is another important factor in manufacturing process. With respect to lubrication dynamic viscosity exponent, this study indicates that influence from dynamic viscosity exponent on cage dynamics is not significant as the prediction.

As a matter of fact, researches in the paper are merely restricted to cage dynamic response. What is to be pointed out is that dynamic response of a cylindrical roller bearing is a complex and interrelated problem among components. Hence, there are many imperfect points in the paper which should be noted here. Preliminary discussions are listed as follows.

(1) The displacements of the cage discussed in the paper are only in $x-y$ plane. During the rotation of the bearing, rollers will inevitably have a certain slippage towards the rings due to insufficient traction friction outside the load-zone, churning moment from lubricant, the friction loss between roller-pocket contact and roller-rib contacts in the actual running process. Roller/raceways contact has not been taken into account in the paper for the sake of simplifying the calculation.

(2) Damping coefficient and contact stiffness involved in the calculation are obtained according to material 


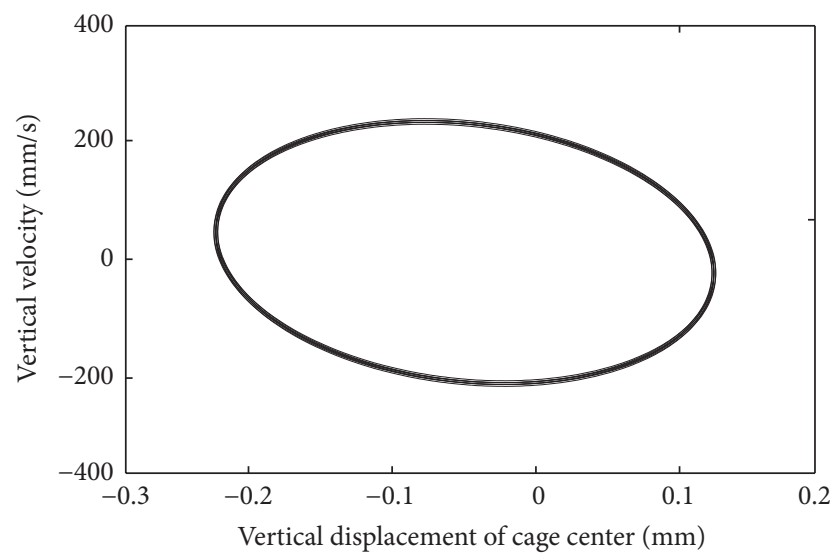

(a) Smaller cage guidance

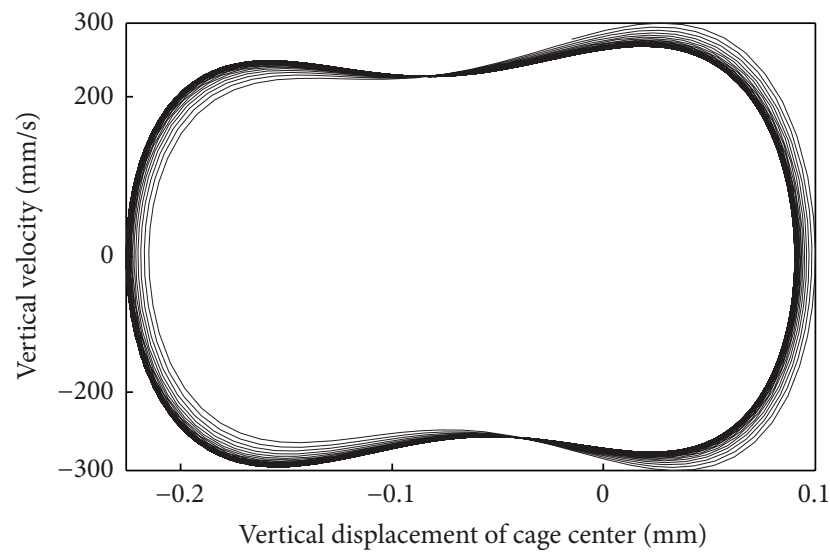

(c) Smaller eccentricity

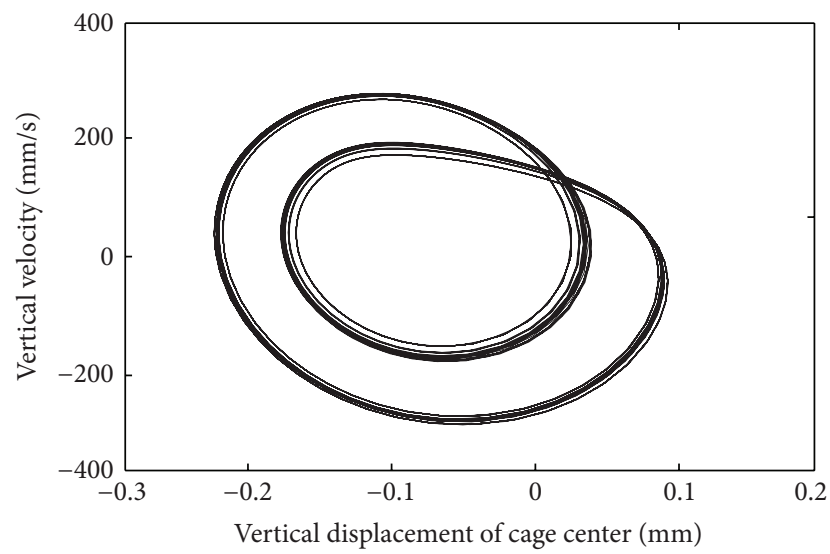

(e) Higher dynamic viscosity exponent

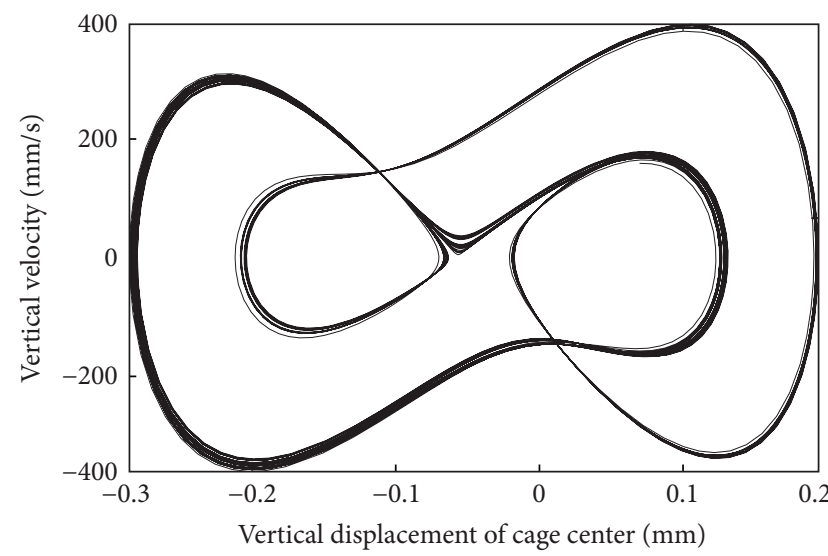

(b) Bigger cage guidance

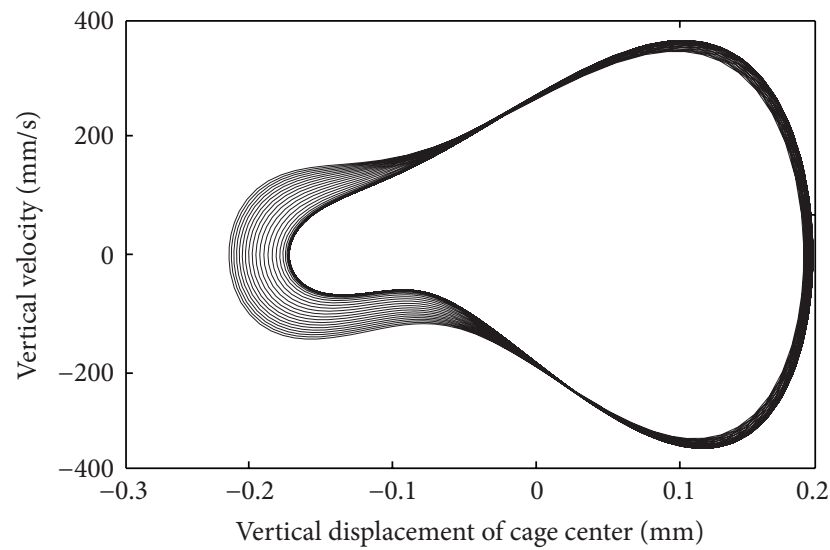

(d) Bigger eccentricity

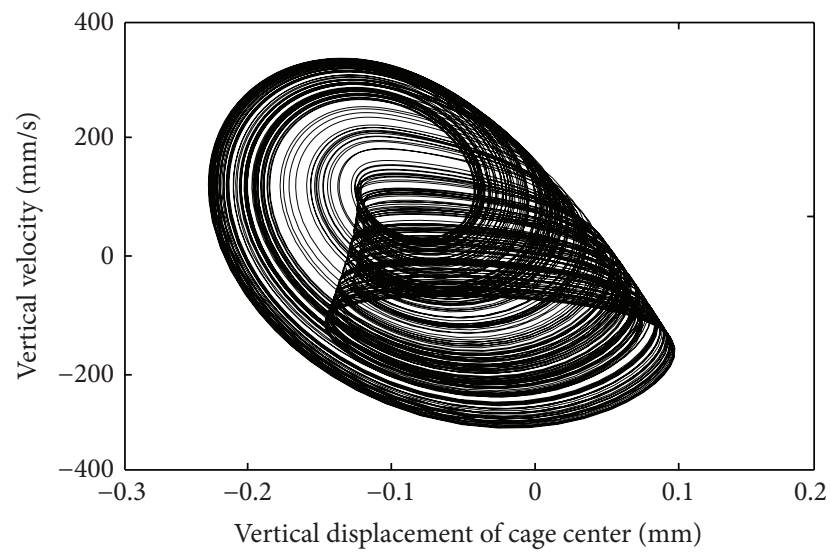

(f) Lower dynamic viscosity exponent

FIgURE 9: Poincaré section points of $Y_{C}$ displacement in vertical direction.

property and geometry characteristic parameters. Actually, the damping coefficient and contact stiffness can be described more accurately by immediate movement and velocity. Namely, contact stiffness is the first-order derivative of immediate movement, and damping coefficient is the first-order derivative of immediate velocity. For the same sake of simplifying calculation, the accurate expressions are not substituted into the equations.
(3) The bearing under study has the outer ring fixed to a rigid support and the inner ring fixed rigidly to the shaft. Bearing house and the shaft are truly nonlinear DOF systems. The influence of bearing house and shaft on cage dynamic is relatively small so it can be simplified in the calculation.

(4) As illustrated in Section 3, cage is driven by the rollers in supporting zone and the rollers are driven by cage 
in nonloaded zone. Driving forces on the total cage are balanced, and they are ignored except the impact forces in switching zones of driven modes.

\section{Symbols}

$\alpha: \quad$ Angle at cage center, in rad

$\dot{\alpha}$ : Angular velocity in tangential direction, in $\mathrm{rad} / \mathrm{s}$

$\ddot{\alpha}$ : Angular acceleration in tangential direction, in $\mathrm{rad} / \mathrm{s}^{2}$

$B:$ Damping coefficient

$C$ : Cage gap, in $\mathrm{mm}$

$d$ : Diameter, in $\mathrm{mm} / \mathrm{s}$

$\Delta:$ Roller gap, in mm

$\delta$ : Deformation, in $\mathrm{mm}$

$e:$ Cage eccentricity, in $\mathrm{mm}$

$\varepsilon$ : Displacement in normal direction, in $\mathrm{mm}$

$\dot{\varepsilon}$ : Velocity in normal direction, in $\mathrm{mm} / \mathrm{s}$

$\ddot{\varepsilon}$ : Acceleration in normal direction, in $\mathrm{mm} / \mathrm{s}^{2}$

$F$ : Load, in N

$f$ : Load caused by motion, in $\mathrm{N}$

$G$ : Clearance, in $\mathrm{mm}$

$\theta$ : Angle, in ${ }^{\circ}$

$K$ : Hertzian stiffness

$l$ : Length, in $\mathrm{mm}$

$M$ : Mass, in $\mathrm{kg}$

$\mu$ : Fluid dynamic viscosity, in $\mathrm{Pa} \cdot \mathrm{s}$

$N$ : Number of the rollers

$v$ : Dimensionless parameter of impact factor

$O:$ Position of the center, in $\mathrm{mm}$

$\dot{O}:$ Velocity vector of the center, in $\mathrm{mm} / \mathrm{s}$

Ö: Acceleration vector of the center, in $\mathrm{mm} / \mathrm{s}^{2}$

$p$ : Hydrodynamic pressure, in $\mathrm{Pa}$

$p_{0}$ : Initial hydrodynamic pressure, in $\mathrm{Pa}$

$Q:$ Hertz stress, in $\mathrm{N} / \mathrm{mm}^{2}$

$R$ : Radius, in $\mathrm{mm}$

$\rho$ : Dimensionless parameter of cage eccentricity to cage gap

$t$ : Time, in sec

$T$ : Pocket clearance, in $\mathrm{mm}$

$v$ : Surface velocity, in $\mathrm{mm} / \mathrm{s}$

$X$ : Coordinate $X$ direction distance, in $\mathrm{mm}$

$x$ : Position in coordinate direction, in $\mathrm{mm}$

$\dot{x}$ : Velocity in $x$ direction, in $\mathrm{mm} / \mathrm{s}$

$\ddot{x}$ : Acceleration in $x$ direction, in $\mathrm{mm} / \mathrm{s}^{2}$

$Y$ : Coordinate $Y$ direction distance, in $\mathrm{mm}$

$y$ : Dimensionless parameter in coordinate direction

$\dot{y}$ : Velocity in $y$ direction, in $\mathrm{mm} / \mathrm{s}$

$\ddot{y}$ : Acceleration in $y$ direction, in $\mathrm{mm} / \mathrm{s}^{2}$

$\omega$ : Rotation speed, in $\mathrm{rad} / \mathrm{s}$.

\section{Subscripts}
a: Axial direction
$\alpha$ : Tangential direction
C: Cage center
c: Cage
cen: Centrifugal

ci: Relative relation between cage and inner ring

co: Relative relation between cage and outer ring

$c w$ : Relative relation between cage and roller

$D$ : Unbalanced gravitational center

e: Normal direction

$f_{d}$ : Added fluid

$g: \quad$ Rib

h: Impact

$i$ : Inner raceway

$j: \quad$ The $j$ th roller

$j_{\text {cen }}$ : Centrifugal force of the $j$ th roller

$m$ : Mean value

O: Rib center

$o$ : Outer raceway

$r$ : Radial direction

$r i$ : Inner raceway on radial direction

ro: Outer raceway on radial direction

$s$ : Polar coordinate

$w$ : Roller

$x: \quad x$ direction

$y: \quad y$ direction.

\section{Conflict of Interests}

The authors declare that there is no conflict of interests regarding the publication of this paper.

\section{Acknowledgments}

The project is supported by National Natural Science Foundation of China (Grant no. 51475144) and the Foundation of Innovation and Research Team of Science and Technology in Universities in Henan Province (Grant no. 13IRTSTHN025).

\section{References}

[1] M. Tiwari, K. Gupta, and O. Prakash, "Dynamic response of an unbalanced rotor supported on ball bearings," Journal of Sound and Vibration, vol. 238, no. 5, pp. 757-779, 2000.

[2] M. L. Adam Jr., Rotating Machinery Vibration-From Analysis to Trouble Shooting, Marcel Dekker, New York, NY, USA, 2001.

[3] N. Aktürk, M. Uneeb, and R. Gohar, "The effects of number of balls and preload on vibrations associated with ball bearings," Journal of Tribology, vol. 119, no. 4, pp. 747-753, 1997.

[4] S. H. Upadhyay, S. C. Jain, and S. P. Harsha, "Non-linear vibration signature analysis of a high-speed rotating shaft due to ball size variations and varying number of balls," Proceedings of the Institution of Mechanical Engineers, Part K: Journal of Multibody Dynamics, vol. 223, no. 2, pp. 83-105, 2009.

[5] N. Aktürk, "The effect of waviness on vibrations associated with ball bearings," Journal of Tribology, vol. 121, no. 4, pp. 667-677, 1999.

[6] S. P. Harsha, K. Sandeep, and R. Prakash, "Non-linear dynamic behaviors of rolling element bearings due to surface waviness," Journal of Sound and Vibration, vol. 272, no. 3-5, pp. 557-580, 2004.

[7] J. Sopanen and A. Mikkola, "Dynamic model of a deep-groove ball bearing including localized and distributed defects. Part 
2: Implementation and results," Proceedings of the Institution of Mechanical Engineers, Part K, vol. 217, no. 3, pp. 213-223, 2003.

[8] S. H. Upadhyay, S. C. Jain, and S. P. Harsha, "Chaotic dynamics of high speed rotating shaft supported by ball bearings due to distributed defects," International Journal of Engineering Science and Technology, vol. 2, no. 10, pp. 5746-5794, 2010.

[9] S. P. Harsha, "Nonlinear dynamic analysis of a high-speed rotor supported by rolling element bearings," Journal of Sound and Vibration, vol. 290, no. 1-2, pp. 65-100, 2006.

[10] C. Villa, J.-J. Sinou, and F. Thouverez, "Stability and vibration analysis of a complex flexible rotor bearing system," Communications in Nonlinear Science and Numerical Simulation, vol. 13, no. 4, pp. 804-821, 2008.

[11] L. Houpert, "CAGEDYN: a contribution to roller bearing dynamic calculations. Part III: experimental validation," ASME Tribology Transactions, vol. 53, no. 6, pp. 848-859, 2010.

[12] S. P. Harsha, "Nonlinear dynamic analysis of rolling element bearings due to cage run-out and number of balls," Journal of Sound and Vibration, vol. 289, no. 1-2, pp. 360-381, 2006.

[13] I. Bercea, S. Cretu, M. Bercea, and D. Olaru, "Simulating rollercage pocket friction in a tapered roller bearing," European Journal of Mechanical and Environmental Engineering, vol. 43, no. 4, pp. 189-194, 1998.

[14] T. Sakaguchi and K. Harada, "Dynamic analysis of cage behavior in a tapered roller bearing," Journal of Tribology, vol. 128, no. 3, pp. 604-611, 2006.

[15] T. A. Harris, Rolling Bearing Analysis, John Wiley \& Sons, 4th edition, 2001.

[16] C. Spiridon, "Machined brass and pressed steel bearing cages: a comparative study," RKB Technical Review, vol. 7, pp. 1-13, 2011.

[17] R. Riccardo, "Chaos theory and some practical applications in technical analysis," in Proceedings of the 11th Annual Conference of the International Federation of Technical Analysts, 1998.

[18] S. Tomoya and U. Kaoru, "Dynamic analysis of cage behavior in a cylindrical roller bearing," NTN Technical Review 71, 2004. 


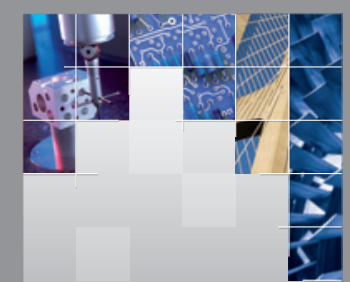

\section{Enfincering}
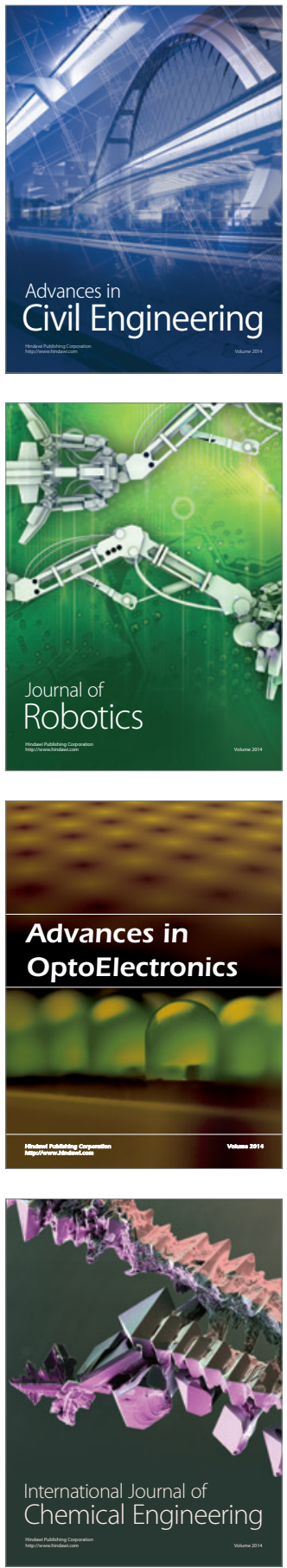

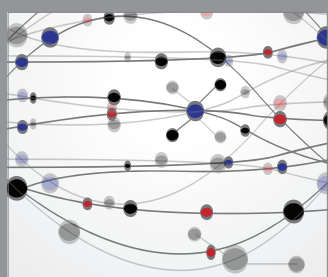

The Scientific World Journal

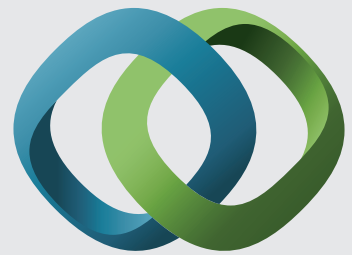

\section{Hindawi}

Submit your manuscripts at

http://www.hindawi.com
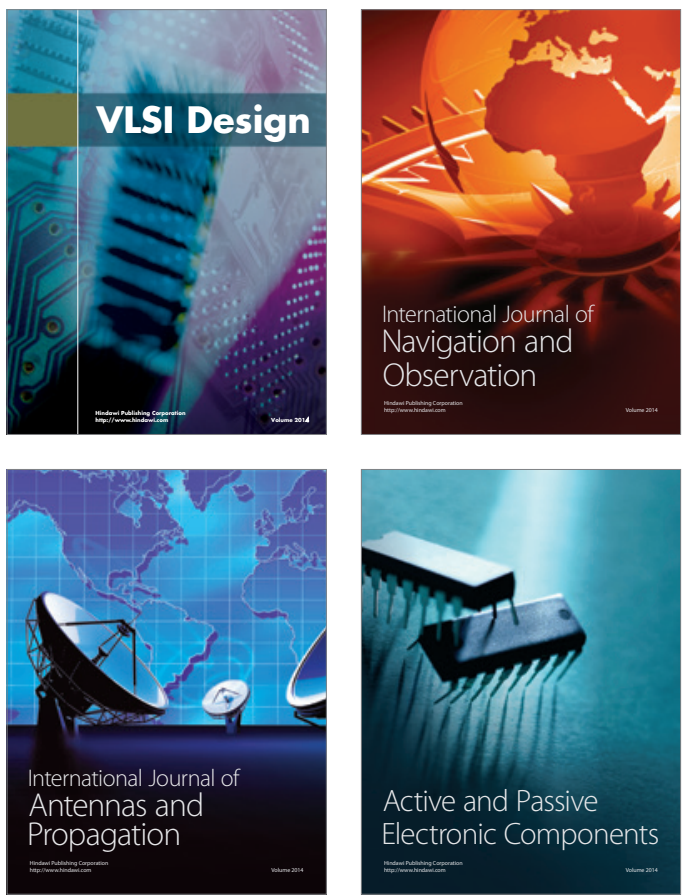
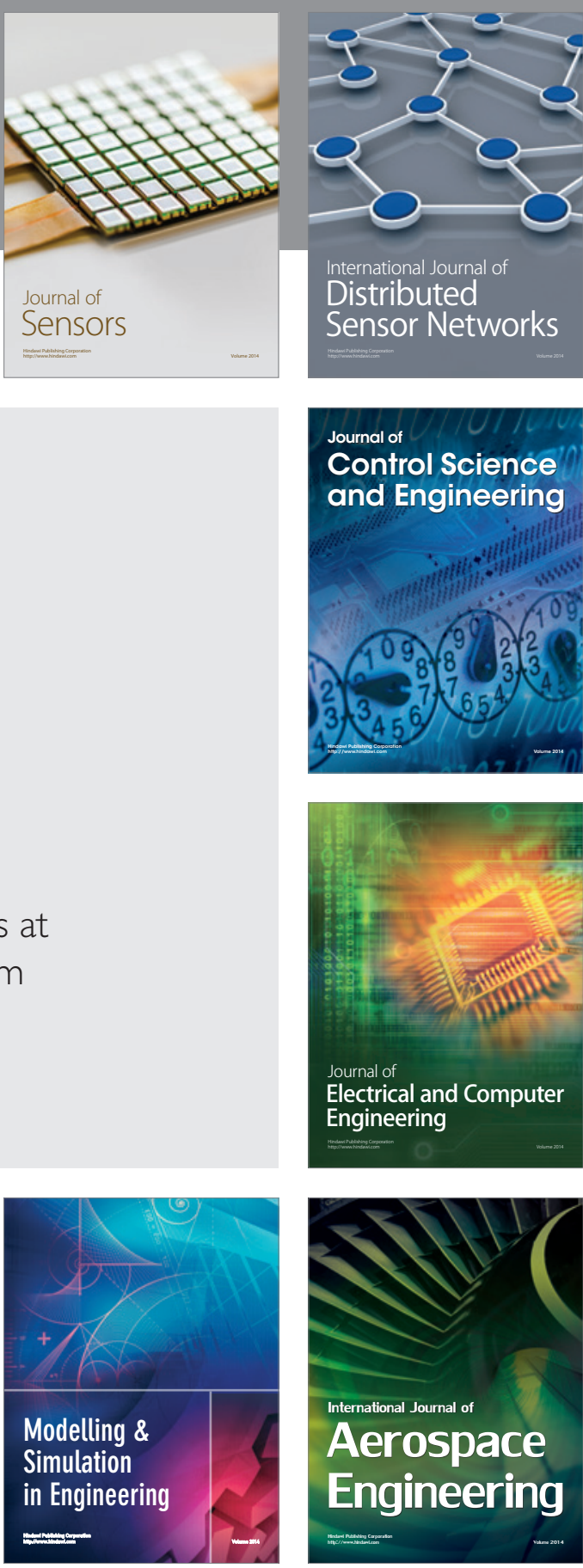

International Journal of

Distributed

Sensor Networks

Journal of

Control Science

and Engineering
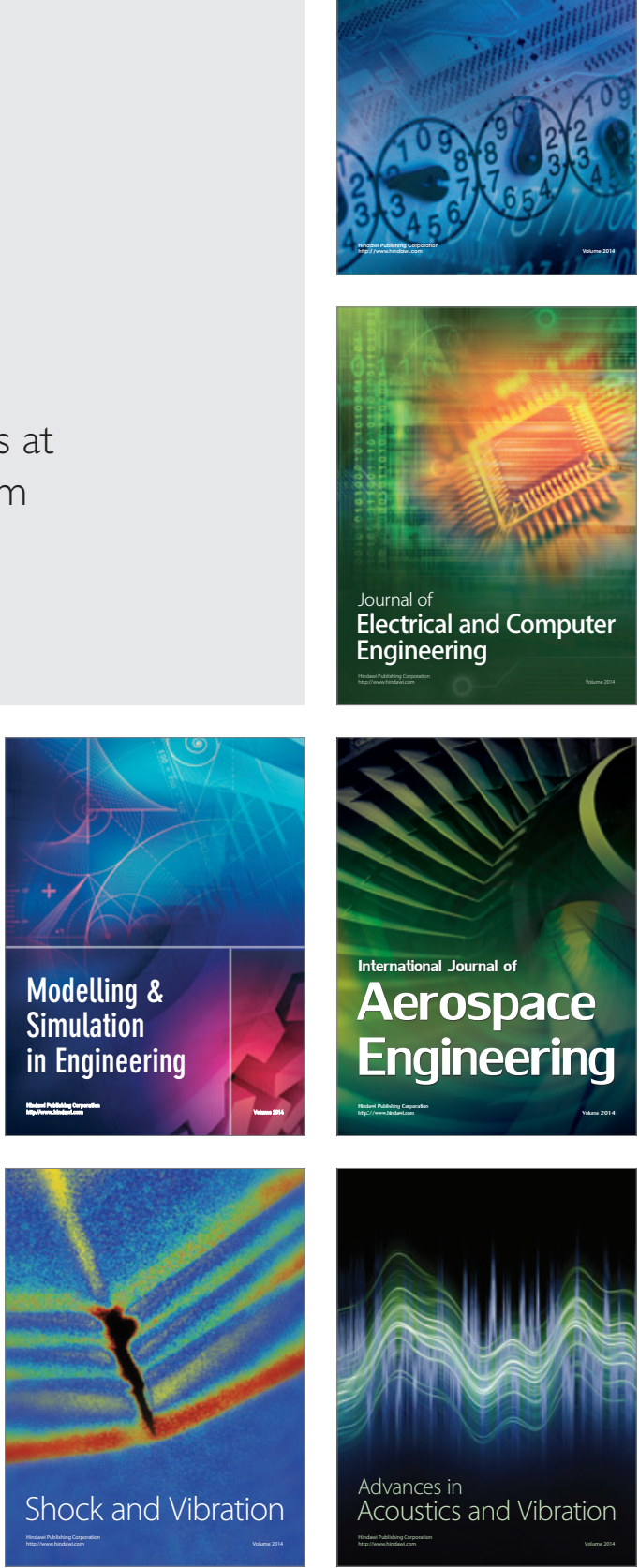